\title{
Language Lateralization Represented by Spatiotemporal Mapping of Magnetoencephalography
}

\author{
N. Tanaka, H. Liu, C. Reinsberger, J.R. Madsen, B.F. Bourgeois, B.A. Dworetzky M.S. Hämäläinen, and S.M. Stufflebeam
}

\begin{abstract}
BACKGROUND AND PURPOSE: Determination of hemispheric language dominance is critical for planning epilepsy surgery. We assess the usefulness of spatiotemporal source analysis of magnetoencephalography for determining language laterality.

MATERIALS AND METHODS: Thirty-five patients with epilepsy were studied. The patients performed a semantic word-processing task during MEG recording. Epochs containing language-related neuromagnetic activity were averaged after preprocessing. The averaged data between 250 and 550 ms after stimulus were analyzed by using dynamic statistical parametric mapping. ROls were obtained in the opercular and triangular parts of the inferior frontal gyrus, superior temporal gyrus, and supramarginal gyrus in both hemispheres. We calculated laterality indices according to 1) dSPM-amplitude method, based on the amplitude of activation in the ROIs, and 2) dSPMcounting method, based on the number of unit dipoles with activation over a threshold in the ROls. The threshold was determined as half of the maximum value in all ROls for each patient. $A L I \geq 0.10$ or $\leq-0.10$ was considered left- or right-hemisphere dominance, respectively; a LI between -0.10 and 0.10 was considered bilateral. All patients underwent an intracarotid amobarbital procedure as part of presurgical evaluation.
\end{abstract}

RESULTS: The dSPM-counting method demonstrated laterality consistent with the IAP in 32 of 35 patients (91.4\%), the remaining 3 (8.6\%) demonstrated bilateral language representation, whereas the dSPM-amplitude method showed 18 (51.4\%) concordant and 17 (48.6\%) bilateral. No laterality opposite to the IAP was found.

CONCLUSIONS: Spatiotemporal mapping of language lateralization with the dSPM-counting method may reduce the necessity for an IAP in as many as $90 \%$ of patients.

ABBREVIATIONS: dSPM = dynamic statistical parametric mapping; IAP = intracarotid amobarbital procedure; $\mathrm{LI}$ = laterality index; $\mathrm{MEG}=$ magnetoencephalography; MPRAGE = magnetization-prepared rapid acquisition of gradient echo

D etermination of hemispheric language dominance (language lateralization) is critical for planning epilepsy surgery. Traditionally, the IAP, also known as the Wada test, is the standard technique used to determine language laterality. It is invasive, requiring the injection of amobarbital (or methohexital) into the

Received March 2, 2012; accepted after revision May 28

From the Athinoula A. Martinos Center for Biomedical Imaging (N.T., H.L., M.S.H., S.M.S.), Massachusetts General Hospital, Charlestown, Massachusetts; Department of Neurology, Brigham and Women's Hospital (C.R., B.A.D.), Boston, Massachusetts; and Departments of Neurosurgery (I.R.M.) and Neurology (B.F.B.), Children's Hospital Boston, Boston, Massachusetts.

This work was supported by the National Institutes of Health (Grants P41-RR14075, R01-NS037462-07), Mental Illness and Neuroscience Discovery Institute, NARSAD Young Investigators Award, and Japan Epilepsy Research Foundation.

Please address correspondence to Naoaki Tanaka, Athinoula A. Martinos Center for Biomedical Imaging, 149 Thirteenth St, Suite 2301, Charlestown, MA 02129; e-mail: naoro@nmr.mgh.harvard.edu

--m Indicates open access to non-subscribers at www.ajnr.org

http://dx.doi.org/10.3174/ajnr.A3233 internal carotid arteries, and carries a risk of stroke and other complications. ${ }^{1-3} \mathrm{MEG}$, a noninvasive neuroimaging procedure, is an established method for language lateralization. ${ }^{4-7}$ MEG detects neural activity with high spatial and temporal resolution when it is recorded in a subject performing language tasks. Previous studies have reported concordance between the IAP and MEG lateralization in presurgical patients with epilepsy, showing positive predictive values of $83 \%-91 \%{ }^{8,9}$

In these studies, MEG data were analyzed by using a conventional single dipole model. ${ }^{4-6}$ This model may be unfavorable in some conditions, especially when the brain activation involves a large region of a neural network. ${ }^{10,11}$ Several studies have reported that the lateralization metrics based on MEG are highly dependent on the dipole selection criteria, and sensitivity ranges from $71 \%$ to $98 \%{ }^{8,9}$ Determination of these criteria may require specialized skill and experience. Spatiotemporally distributed source models, such as minimum norm estimates and dSPM, provide good source localization over extended sources. ${ }^{12,13}$ These 
Patient profile and laterality indices

\begin{tabular}{|c|c|c|c|c|}
\hline Patient & Age/Sex & Diagnosis & LI-Amplitude & LI-Counting \\
\hline 1 & $16 / F$ & Lt TLE & 0.27 & 0.88 \\
\hline 2 & $13 / F$ & Lt FLE & $-0.03^{a}$ & 0.30 \\
\hline 3 & $15 / \mathrm{M}$ & Lt TLE & $0.05^{\mathrm{a}}$ & 0.58 \\
\hline 4 & $15 / F$ & Rt TLE & $0.02^{\mathrm{a}}$ & 0.14 \\
\hline 5 & $34 / F$ & Lt TLE & 0.54 & 1.00 \\
\hline 6 & $20 / \mathrm{F}$ & Rt TLE & 0.12 & 0.98 \\
\hline 7 & $19 / \mathrm{M}$ & Rt FLE & 0.12 & 0.89 \\
\hline 8 & $15 / \mathrm{M}$ & Rt PLE & 0.24 & 0.68 \\
\hline 9 & $17 / F$ & Lt TLE & 0.11 & 0.68 \\
\hline 10 & $9 / \mathrm{F}$ & Lt TLE & $0.02^{\mathrm{a}}$ & 0.28 \\
\hline 11 & $14 / \mathrm{M}$ & Rt FLE & $-0.06^{\mathrm{a}}$ & $-0.08^{a}$ \\
\hline 12 & $14 / F$ & Lt TLE & 0.15 & 0.62 \\
\hline 13 & $16 / F$ & Lt TLE & 0.16 & 0.63 \\
\hline 14 & $28 / \mathrm{M}$ & Lt FLE & $0.08^{a}$ & 0.16 \\
\hline 15 & $18 / \mathrm{M}$ & Lt OLE & $0.02^{\mathrm{a}}$ & $0.05^{\mathrm{a}}$ \\
\hline 16 & $21 / M$ & Lt TLE & $0.01^{\mathrm{a}}$ & 0.33 \\
\hline 17 & $38 / \mathrm{M}$ & Rt TLE & $0.04^{a}$ & 0.62 \\
\hline 18 & $30 / \mathrm{M}$ & Rt TLE & $-0.07^{\mathrm{a}}$ & 0.15 \\
\hline 19 & $28 / F$ & Rt TLE & 0.26 & 0.99 \\
\hline 20 & $42 / F$ & Rt TLE & 0.10 & 0.15 \\
\hline 21 & $50 / \mathrm{M}$ & Lt TLE & 0.10 & $-0.04^{\mathrm{a}}$ \\
\hline 22 & $18 / \mathrm{M}$ & Rt TLE & 0.12 & 0.22 \\
\hline 23 & $59 / \mathrm{M}$ & Lt TLE & 0.17 & 0.88 \\
\hline 24 & $26 / \mathrm{M}$ & Lt TLE & $-0.08^{a}$ & 0.10 \\
\hline 25 & $44 / \mathrm{M}$ & Lt TLE & $0.00^{\mathrm{a}}$ & 0.23 \\
\hline 26 & $15 / \mathrm{M}$ & Lt TLE & $0.07^{\mathrm{a}}$ & 0.23 \\
\hline 27 & $23 / \mathrm{M}$ & Rt TLE & 0.11 & 0.47 \\
\hline 28 & $18 / F$ & Lt TLE & 0.11 & 0.20 \\
\hline 29 & $28 / \mathrm{F}$ & Rt TLE & 0.11 & 0.59 \\
\hline 30 & $26 / \mathrm{F}$ & Rt TLE & $0.06^{\mathrm{a}}$ & 0.13 \\
\hline 31 & $30 / F$ & Lt TLE & 0.11 & 0.56 \\
\hline 32 & $14 / F$ & Rt OLE & 0.24 & 1.00 \\
\hline $33^{b}$ & $15 / \mathrm{M}$ & Lt FLE & $-0.02^{\mathrm{a}}$ & -0.17 \\
\hline $34^{\mathrm{b}}$ & $43 / \mathrm{M}$ & Lt TLE & $-0.06^{\mathrm{a}}$ & -0.13 \\
\hline $35^{b}$ & $12 / \mathrm{M}$ & Lt TLE & $-0.09^{\mathrm{a}}$ & -0.33 \\
\hline
\end{tabular}

Note:-LI indicates laterality index; F, female; M, male; Rt, right; Lt, left; TLE, temporal lobe epilepsy; FLE, frontal lobe epilepsy; PLE, parietal lobe epilepsy; OLE, occipital lobe epilepsy.

${ }^{a}$ MEG-derived language lateralization is not consistent with IAP.

${ }^{b}$ Right-hemisphere language dominance in IAP.

estimate the current of unit dipoles distributed in the numerous cortical patches, creating dynamic activation maps on the cortex. A few studies have applied spatiotemporal source models to analyzing the language-related MEG data. ${ }^{14,15}$ However, the clinical usefulness of an anatomically constrained dSPM remains unclear.

The purpose of this study was to assess the usefulness of a spatiotemporal source analysis of MEG data for determining language laterality in patients with medically refractory epilepsy. From a practical perspective, we sought semiautomated procedures without the need for subjective interactions such as manually identifying MEG sources, sensors, and signals. We calculated the LI in 2 different ways based on dSPM, and compared these with the laterality obtained from the IAP.

\section{MATERIALS AND METHODS}

\section{Patients}

We studied 35 patients (16 female, 19 male; mean age, 24.0 years; Table) with intractable epilepsy undergoing presurgical evaluation. MEG was performed as a part of this clinical evaluation, and we analyzed all data retrospectively. Three patients (patients 33-
35) showed right-hemisphere language dominance in the IAP, and the rest showed left-hemisphere dominance.

\section{Control Group}

We studied a control group to validate the capability of language lateralization by using the procedure performed in the patients. The control group consisted of 25 healthy, right-handed subjects (7 male, 18 female; mean age, 24.2 years). The study was approved by the Massachusetts General Hospital institutional review board.

\section{Data Acquisition}

MEG was recorded with a 306-channel (204 planar gradiometers and 102 magnetometers), whole-head MEG system in a magnetically shielded chamber. The sampling rate was $1 \mathrm{kHz}$ (patients 1-9) or $600 \mathrm{~Hz}$ (patients 10-35). Data were bandpass filtered between 0.1 and $200 \mathrm{~Hz}$. For the source analysis, the data were further low-pass filtered with a cutoff frequency of $40 \mathrm{~Hz}$. In all patients, high-resolution 3T anatomic MR imaging data were acquired with magnetization-prepared rapid acquisition of gradient echo $(\mathrm{TE}=3.37 \mathrm{~ms}$; $\mathrm{TR}=2000 \mathrm{~ms}$; voxel size $=1 \times 1 \times 1 \mathrm{~mm})$.

\section{Stimuli and Tasks}

During the MEG recording, patients performed a semantic decision task in which 160 English nouns were presented visually. They were asked to decide whether each word was abstract (eg, "freedom") or concrete (eg, "apple") and to press different buttons depending on their decision (Fig 1A). Each word was presented for $1000 \mathrm{~ms}$, and the interstimulus interval was $2000 \mathrm{~ms}$. Total recording time was 8 minutes for each patient.

\section{Preprocessing}

Each patient's cortical surface was reconstructed from the MPRAGE MR imaging data, and anatomic parcellation of the cortical areas was obtained by using FreeSurfer (http://surfer. nmr.mgh.harvard.edu) ${ }^{16,17}$ (Fig 1C, $-D$ ). For MEG data, epochs with a time window of $500 \mathrm{~ms}$ before stimulus and $1000 \mathrm{~ms}$ after stimulus were collected (Fig 1A). Each epoch was examined visually for artifact rejection. The MEG data containing adequate epochs were processed with a temporally extended signal space separation method for further reduction of artifacts. ${ }^{18,19}$ After the preprocessing, these epochs were averaged for each patient. Thus, the averaged MEG data contain neuromagnetic fields evoked by repetitive language stimuli (Fig $1 B$ ). The source activity was then estimated based on the averaged data (Fig $1 E$ ).

\section{Source Analysis}

Spatiotemporal source distribution was calculated by using dSPM. ${ }^{13}$ In this method, an anatomically constrained linear estimation approach was applied, assuming the sources are distributed on the cortical surface. ${ }^{20}$ The forward solution, which models the signal pattern generated by a unit dipole at each location on the cortical surface, was calculated by using a single-layer boundary elemental method. ${ }^{21,22}$ The surface was tessellated with 5120 triangles; adequate numeric accuracy was provided by using FreeSurfer. ${ }^{16,17,23-26}$ The activation at each vertex of the cortical surface was mapped by using a noise-normalized minimum norm estimate. ${ }^{12,20}$ Thus, the spatiotemporal source distribution maps 


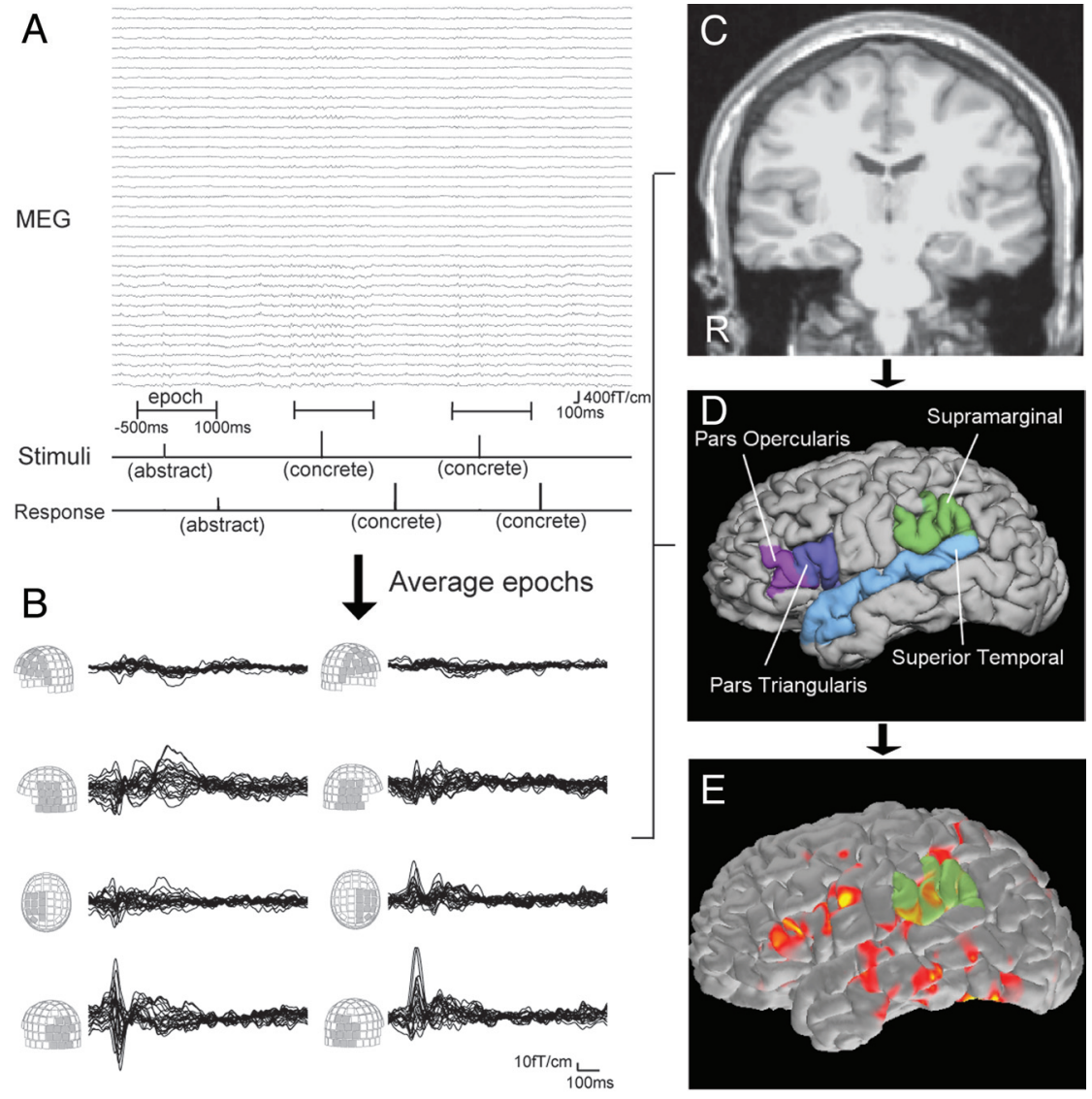

FIG 1. A, Schematic representation of language MEG processing. Stimuli are visually presented as "abstract" or "concrete" words (Stimuli line). Patient responses are also recorded (Response line). Using stimuli as a trigger, epochs from $-500 \mathrm{~ms}$ to $1000 \mathrm{~ms}$ are averaged. $B$, Waveforms of the averaged MEG from 0 ms to 1000 ms. Waveforms of each MEG sensor group are superimposed. Language activation is seen around $250-550 \mathrm{~ms}$ in the frontal, temporal, and parietal sensors. MPRAGE MR imaging $(C)$ provides the cortical surface of each patient by reconstruction processing. This procedure also gives anatomic parcellation of the cortex $(D)$. Four ROIs per each hemisphere are used for calculating LI. E, Spatiotemporal source distribution of the language MEG is mapped on the cortical surface by using dSPM. The value of activation in ROls is extracted from dSPM.

were obtained at each time point (Fig $1 E)$. We evaluated the maps within a time window of 250-550 ms.

\section{Laterality Index}

LI was calculated by 2 different methods with the use of dSPM based on the source distribution in several ROIs. The Wernicke and Broca areas are important for representing receptive and expressive language function, whereas various other regions, such as dorsolateral prefrontal cortex and primary motor cortex, participate in language processing. ${ }^{27,28}$ Presurgical evaluation usually requires mapping of the essential language areas and not just the participating areas. We selected ROIs based on a priori knowledge of the language network, ${ }^{14,15}$ including opercular and triangular parts of the inferior frontal gyrus, superior temporal gyrus, and supramarginal gyrus in both hemispheres (Fig 1D). These cortical areas were created by an automated cortical parcellation algorithm by using FreeSurfer and were visually inspected to avoid erroneous surface reconstruction. LI values range from -1.00 to 1.00 , and a LI $\geq 0.10$ or $\leq-0.10$ was considered left- or righthemisphere dominance, respectively; a LI between -0.10 and 0.10 was considered bilateral.

\section{dSPM Amplitude Method}

We averaged source waveforms within each region of interest. The sum of amplitude in a time window of $250-550 \mathrm{~ms}$ was obtained for all ROIs. LI-amplitude was defined as $(L-R) /(L+R)$, where $L$ and $R$ represent the amplitudes in left- and right-hemisphere ROIs, respectively (Fig 2A).

\section{dSPM Counting Method}

In each ROI, we counted the number of unit dipoles the activation of which exceeded a threshold in the time window of 250-550 ms. The threshold was determined as half of the maximum value over all ROIs. LI-counting was defined as $(L-$ $R) /(L+R)$, where $L$ and $R$ represent the total number of unit dipoles counted in left- and right-hemisphere ROIs, respectively (Fig 2B).

\section{RESULTS}

Table 1 provides an overview of the results for all patients. With the dSPM-amplitude method, 18 of 35 (51.4\%) patients showed language representation consistent with IAP results. Seventeen patients $(48.6 \%)$ had bilateral representation. In the dSPM-counting method, 32 (91.4\%) were concordant and $3(8.6 \%)$ were bilateral. LI-counting was more consistent with IAP lateralization than LI-amplitude. We estimated the concordance between the lateralization results of MEG and the IAP regarding the laterality in the epileptogenic hemisphere. ${ }^{8}$ In LI-amplitude, the sensitivity, specificity, positive predictive value, and negative predictive value were $100 \%, 53 \%, 69 \%$, and $100 \%$ when bilateral representation was classified into the positive group for both hemispheres. In LI-counting, these values were 100\%, 94\%, $95 \%$, and $100 \%$.

In the control group, LI-amplitude showed left-sided language predominance in 11 of $25(44.0 \%)$ subjects. Fourteen (56.0\%) were bilateral. In LI-counting, 23 of $25(92.0 \%)$ were left, and 2 $(8.0 \%)$ were bilateral.

\section{DISCUSSION}

In this study, we introduced 2 procedures for language lateralization by using spatiotemporal source analysis of MEG, and we compared the results by using an IAP. Our results demonstrated that the language laterality estimated by using the dSPM-counting method had a good concordance with the IAP.

Our procedure included a semantic decision task for language MEG acquisition. A semantic task has been performed in prior studies. ${ }^{7,15}$ Binder et al ${ }^{29}$ reported that the semantic task strongly activated the language network of the brain with fMRI. LI-count- 

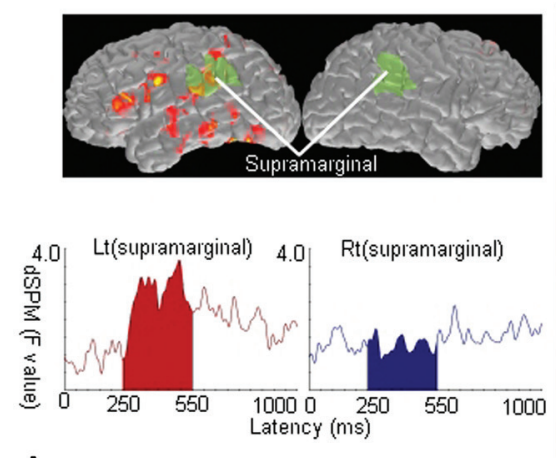

A

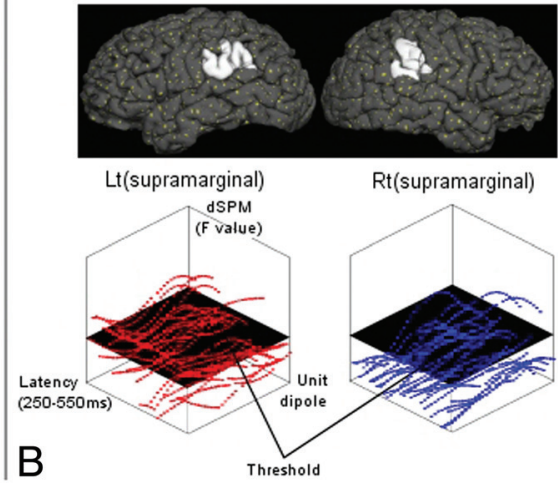

FIG 2. Schematic representation for calculating the $L I$. $L I$ is calculated by the formula $(L-R) /(L$ $+R$ ), where $L$ and $R$ represent parameters obtained from the source distribution within a time window of $250-550 \mathrm{~ms}$ in the left and right ROIs, respectively. A, dSPM-amplitude method. The source waveform averaged in each region of interest is extracted from dSPM. $L$ and $R$ are the sum of these waveforms in left and right ROls. The figure shows an example of processing in a pair of ROIs (supramarginal gyri). The dSPM (top) provides source waveforms averaged within the left and right supramarginal gyrus (bottom). B, dSPM-counting method. In dSPM, each unit dipole (top; yellow dots) has a value of activation. Within each region of interest (eg, the left and right supramarginal gyrus; top), the values of numerous unit dipoles are obtained at each time point (bottom). The number of unit dipoles with a value over a threshold is counted. $L$ and $R$ are the total number of unit dipoles counted in left and right ROIs.

ing in our control group showed a high proportion of left predominance, consistent with a previous report ${ }^{30}$ suggesting that the task paradigm is appropriate for language lateralization.

Several studies have shown a good concordance of language laterality between MEG and the IAP by using a single dipole model. ${ }^{8,9}$ These studies used optimized dipole selection criteria $^{6,8,9}$ and optimized sensor selection ${ }^{4,8,31,32}$ for calculating dipoles adequately demonstrating language-related activity. Although highly accurate, this process is rather time consuming and requires significant skill. Several researchers have demonstrated that the optimization may be important for determining laterality by MEG. ${ }^{33}$ In theory, dSPM can represent all the magnetic fields by applying a distributed source model. ${ }^{13,20}$ Therefore, dSPM analysis does not have a concept of the selection criteria of the adequate sources. In addition, source waveforms, or values of activation, can easily be extracted from any cortical regions of interest on dSPM based on cortical parcellation. Our LI calculation procedures do not need manual signal selection at a sensor level. By using an objective process in the whole procedure, dSPM may be a convenient method to obtain the language laterality.

LI-amplitude tended to show bilateral representation more than LI-counting, though no patients showed laterality opposite to the IAP. This tendency was also observed in the control group. The dSPM-amplitude method reflects mainly the strength of the language-related neuromagnetic activity. Bilateral representation suggests that the language task activated cortices in both hemispheres. The dSPM-counting method, however, was more concordant with the IAP than the dSPM-amplitude method. It represents the size of activated cortex during the language processing. Thus, these LIs may provide different lateralization results because they reflect different aspects of language-related neuromagnetic activities. Our findings suggest that language lateralization may be more correlated with the size of the activated cortex than with the strength of activation. Previous MEG studies have demonstrated language laterality by counting the number of strong language lateralization.

single-current equivalent dipoles, ${ }^{8,32,34}$ whereas other studies observed bilateral activation associated with language function. ${ }^{15,35}$ None of these studies, however, compared the counting- and amplitude-based methods directly in the same mapping procedure. Language fMRI studies often calculate LIs based on the number of activated voxels over a threshold, ${ }^{36,37}$ but Suarez et $\mathrm{al}^{38}$ have reported that the threshold-dependent approach may demonstrate unfavorable lateralization results. Pang et $\mathrm{al}^{39}$ have compared MEG and fMRI lateralization by using a voxel-counting procedure and demonstrated concordance. Direct comparison between the laterality determined by the size of activated cortex and activation amplitude in the same mapping procedure of both MEG and fMRI would be necessary to determine which approach contributes more to the

Although LI-counting showed high concordance of laterality with the IAP, 3 discordant cases were observed. Patient movement or dental hardware metallic artifacts contaminated the MEG of these patients during visual inspection. Our MEG preprocessing consisted of 2 steps that used manual artifact rejection and signal space separation; lateralization, however, may be difficult in cases such as these with excessive artifacts. In addition, Doss et $\mathrm{al}^{34}$ described fundamental reasons for discordance; MEG is a brain activation paradigm, whereas the IAP suppresses brain activity, and the tasks in these 2 procedures are often very different.

In the dSPM-counting method, we used a threshold determined by half of the maximum value of activation in the ROIs. The best procedure for obtaining an appropriate threshold in dSPM has not been determined. ${ }^{11}$ Investigating the statistical distribution of activation values may be useful for resolving this issue.

Our dSPM procedure uses automated cortical reconstruction and parcellation. Surface-based cortical analysis based on this technique demonstrates structural abnormality of cortical thickness and blurred gray-white matter boundaries. ${ }^{40}$ Therefore, any cortical lesions could theoretically affect the surface reconstruction and source distribution, though there were no gross changes. Further investigation will be necessary to estimate the effect of cortical lesions on the source estimates.

The small number of patients with right-sided language laterality in the IAP is a limitation of this study. Further studies that include more patients with right-dominant and bilateral language representation in the IAP will reduce any selection bias. In this study, our MEG protocol requires only an 8-minute recording session for language lateralization. Including this noninvasive language evaluation in the presurgical work-up would be beneficial and might provide an opportunity to skip an IAP in $90 \%$ of patients.

AJNR Am J Neuroradiol 34:558-63 Mar 2013 www.ajnr.org 


\section{CONCLUSIONS}

Our results suggest that spatiotemporal source analysis of language-related MEG is a rapid, noninvasive, objective method for determining language laterality. Compared with the LI-amplitude method, LI-counting is more concordant with the IAP, indicating that IAP results have more correlation with the size of activated cortex during language processing than with the strength of activation. Spatiotemporal mapping of language lateralization with the dSPM-counting method may reduce the necessity for an IAP in as many as $90 \%$ of patients.

Disclosures: Naoaki Tanaka—RELATED: Grant: Japan Epilepsy Research Foundation. Hesheng Liu—RELATED: Grant: National Institutes of Health (money paid to institution), Comments: Hesheng Liu is supported by National Institutes of Health grant 1K25NS069805. Claus Reinsberger-UNRELATED: Grants/Grants Pending: Epilepsy Foundation, ACNS. Blaise Bourgeois-UNRELATED: Consultancy: Upsher-Smith, Comments: One-time consulting regarding potential clinical trial of intranasal midazolam for acute seizures; Grants/Grants Pending: Lundbeck; Royalties: Cambridge Press, Comments: Published book on Antiepileptic Drugs. Barbara Dworetzky—UNRELATED: Consultancy: SleepMed, Comments: EEG consulting, clinical care; Other: Best Doctors, Consultant. Matti Hämäläinen—RELATED: Grant: National Institutes of Health (money paid to institution), Comments: Grant support from National Institute of Biomedical Imaging and Bioengineering and National Center for Research Resources; Fees for Participation in Review Activities such as Data Monitoring Boards, Statistical Analysis, Endpoint Committees, and the Like: Grant reviews for National Institutes of Health; UNRELATED: Grants/Grants Pending: National Institutes of Health, NSF. Steven Stufflebeam—RELATED: Grant: National Institutes of Health (money paid to institution), Comments: Several National Institutes of Health grants acknowledged in the manuscript.

\section{REFERENCES}

1. Dion JE, Gates PC, Fox AJ, et al. Clinical events following neuroangiography: a prospective study. Stroke 1987;18:997-1004

2. Snyder PJ, Harris LJ. The intracarotid amobarbital procedure: an historical perspective. Brain Cogn 1997;33:18-32

3. de Paola L, Mader MJ, Germiniani FM, et al. Bizarre behavior during intracarotid sodium amytal testing (WADA test): are they predictable? Arq Neuropsiquiatr 2004;62:444-48

4. Simos PG, Breier JI, Zouridakis G, et al. Assessment of functional cerebral laterality for language using magnetoencephalography. J Clin Neurophysiol 1998;15:364-72

5. Papanicolaou AC, Simos PG, Breier JI, et al. Magnetoencephalographic mapping of the language-specific cortex. J Neurosurg 1999; 90:85-93

6. Breier JI, Simos PG, Zouridakis G, et al. Lateralization of cerebral activation in auditory verbal and non-verbal memory tasks using magnetoencephalography. Brain Topogr 1999;12:89-97

7. Kamada K, Sawamura Y, Takeuchi F, et al. Expressive and receptive language areas determined by a non-invasive reliable method using functional magnetic resonance imaging and magnetoencephalography. Neurosurgery 2007;60:296-305

8. Papanicolaou AC, Simos PG, Castillo EM, et al. Magnetocephalography: a noninvasive alternative to the Wada procedure. J Neurosurg 2004;100:867-76

9. Merrifield WS, Simos PG, Papanicolaou AC, et al. Hemispheric language dominance in magnetoencephalography: sensitivity, specificity, and data reduction techniques. Epilepsy Behav 2007;10:120-28

10. Kobayashi K, Yoshinaga H, Ohtsuka Y, et al. Dipole modeling of epileptic spikes can be accurate or misleading. Epilepsia 2005;46: 397-408

11. Tanaka N, Cole AJ, von Pechmann D, et al. Dynamic statistical parametric mapping for analyzing ictal magnetoencephalographic spikes in patients with intractable frontal lobe epilepsy. Epilepsy Res 2009;85:279-86

12. Hämäläinen MS, Ilmoniemi RJ. Interpreting magnetic fields of the brain: minimum norm estimates. Med Biol Eng Comput 1994;32: 35-42

13. Dale AM, Liu AK, Fischl BR, et al. Dynamic statistical parametric mapping: combining fMRI and MEG for high-resolution imaging of cortical activity. Neuron 2000;26:55-67

14. Bowyer SM, Moran JE, Weiland BJ, et al. Language laterality determined by MEG mapping with MR-FOCUSS. Epilepsy Behav 2005;6: 235-41

15. McDonald CR, Thesen T, Hagler DJ Jr., et al. Distributed source modeling of language with magnetoencephalography: application to patients with intractable epilepsy. Epilepsia 2009;50:2256-66

16. Dale AM, Fischl B, Sereno MI. Cortical surface-based analysis. I. Segmentation and surface reconstruction. Neuroimage 1999;9: 179-94

17. Fischl B, Sereno MI, Dale AM. Cortical surface-based analysis. II: Inflation, flattening, and a surface-based coordinate system. Neuroimage 1999;9:195-207

18. Taulu S, Kajola M, Simola J. Suppression of interference and artifacts by the signal space separation method. Brain Topogr 2004;16: 269-75

19. Taulu S, Simola J. Spatiotemporal signal space separation method for rejecting nearby interference in MEG measurements. Phys Med Biol 2006;51:1759-68

20. Dale AM, Sereno MI. Improved localization of cortical activity by combining EEG and MEG with MRI cortical surface reconstruction: a linear approach. J Cogn Neurosci 1993;5:162-76

21. Hämäläinen MS, Sarvas J. Realistic conductivity geometry model of the human head for interpretation of neuromagnetic data. IEEE Trans Biomed Eng 1989;36:165-71

22. Oostendorp TF, van Oosterom A. Source parameter estimation in inhomogeneous volume conductors of arbitrary shape. IEEE Trans Biomed Eng 1989;36:382-91

23. Crouzeix A, Yvert B, Bertrand O, et al. An evaluation of dipole reconstruction accuracy with spherical head model and realistic head models in MEG. Clin Neurophysiol 1999;110:2176-88

24. Fuchs M, Wagner M, Kastner J. Boundary element method volume conductor models for EEG source reconstruction. Clin Neurophysiol 2001;112:1400-07

25. Tarkiainen A, Liljeström M, Seppä M, et al. The 3D topography of MEG source localization accuracy: effects of conductor model and noise. Clin Neurophysiol 2003;114:1977-92

26. de Jongh A, de Munck JC, Gonçalves SI, et al. Differences in MEG/ EEG epileptic spike yields explained by regional differences in signal-to-noise ratios. J Clin Neurophysiol 2005;22:153-58

27. Démonet JF, Thierry G, Cardebat D. Renewal of the neurophysiology of language: functional neuroimaging. Physiol Rev 2005;85: 49-95

28. Stufflebeam SM. Clinical magnetoencephalography for neurosurgery. Neurosurg Clin N Am 2011;22:153-67

29. Binder JR, Westbury CF, McKiernan KA, et al. Distinct brain systems for processing concrete and abstract concepts. J Cogn Neurosci 2005 ; 17:905-17

30. Knecht S, Dräger B, Deppe $M$, et al. Handedness and hemispheric language dominance in healthy humans. Brain 2000;123:2512-18

31. Simos PG, Sarkari S, Castillo EM, et al. Reproducibility of measures of neurophysiological activity in Wernicke's area: a magnetic source imaging study. Clin Neurophysiol 2005;116:2381-91

32. Papanicolaou AC, Pazo-Alvarez P, Castillo EM, et al. Functional neuroimaging with MEG: normative language profiles. Neuroimage 2006;33:326-42

33. Passaro AD, Rezaie R, Moser DC, et al. Optimizing estimation of hemispheric dominance for language using magnetic source imaging. Brain Res 2011;1416:44-50

34. Doss RC, Zhang W, Risse GL, et al. Lateralizing language with magnetic source imaging: validation based on the Wada test. Epilepsia 2009;50:2242-48

35. Hirata M, Goto T, Barnes G, et al. Language dominance and map- 
ping based on neuromagnetic oscillatory changes: comparison with invasive procedures. J Neurosurg 2010;112:528-38

36. Binder JR, Swanson SJ, Hammeke TA, et al. Determination of language dominance using functional MRI: a comparison with the Wada test. Neurology 1996;46:978-84

37. Binder JR, Swanson SJ, Hammeke TA, et al. A comparison of five fMRI protocols for mapping speech comprehension systems. Epilepsia 2008;49:1980-97

38. Suarez RO, Whalen S, Nelson AP, et al. Threshold-independent functional MRI determination of language dominance: a validation study against clinical gold standards. Epilepsy Behav 2009;16: 288-97

39. Pang EW, Wang F, Malone M, et al. Localization of Broca's area using verb generation tasks in the MEG: validation against fMRI. Neurosci Lett 2011;490:215-19

40. Thesen T, Quinn BT, Carlson C, et al. Detection of epileptogenic cortical malformations with surface-based MRI morphometry. PLoS One 2011;6:e16430 